\title{
Preoperative localization of seizure onset zones by magnetic source imaging, EEG-correlated functional MRI, and their combination
}

\author{
*Assaf Berger, MD, ${ }^{1,7}$ Noa Cohen, MSc, ${ }^{2,7}$ Firas Fahoum, MD, MSc, ${ }^{3,7}$ \\ Mordekhay Medvedovsky, MD, PhD, ${ }^{4,8}$ Aaron Meller, BSc, ${ }^{2,7}$ Dana Ekstein, MD, PhD, ${ }^{4,8}$ \\ Mony Benifla, MD, ${ }^{5,9}$ Orna Aizenstein, MD, ${ }^{6,7}$ Itzhak Fried, MD, PhD, ${ }^{1,7}$ Tomer Gazit, PhD, ${ }^{2,7}$ and \\ Ido Strauss, MD, PhD ${ }^{1,7}$
}

${ }^{1}$ Department of Neurosurgery, ${ }^{2}$ Sagol Brain Institute, ${ }^{3}$ Department of Neurology, and ${ }^{6}$ Department of Radiology, Tel Aviv Medical Center, Tel Aviv; " ${ }^{4}$ Department of Neurology, Hadassah Medical Center, Jerusalem; ${ }^{5}$ Department of Neurosurgery, Rambam Health Care Campus, Haifa; ${ }^{7}$ Sackler Faculty of Medicine, Tel-Aviv University, Tel Aviv; ${ }^{8} \mathrm{Hebrew}$ University Hadassah Medical School, Jerusalem; and ${ }^{9}$ Rappaport Faculty of Medicine-Technion, Haifa, Israel

OBJECTIVE Preoperative localization of seizure onset zones (SOZs) is an evolving field in the treatment of refractory epilepsy. Both magnetic source imaging (MSI), and the more recent EEG-correlated functional MRI (EEG-fMRI), have shown applicability in assisting surgical planning. The purpose of this study was to evaluate the capability of each method and their combination in localizing the seizure onset lobe (SL).

METHODS The study included 14 patients who underwent both MSI and EEG-fMRI before undergoing implantation of intracranial EEG (icEEG) as part of the presurgical planning of the resection of an epileptogenic zone (EZ) during the years 2012-2018. The estimated location of the SL by each method was compared with the location determined by icEEG. Identification rates of the SL were compared between the different methods.

RESULTS MSI and EEG-fMRI showed similar identification rates of SL locations in relation to icEEG results $(88 \% \pm$ $31 \%$ and $73 \% \pm 42 \%$, respectively; $p=0.281$ ). The additive use of the coverage lobes of both methods correctly identified $100 \%$ of the SL, significantly higher than EEG-fMRI alone $(p=0.039)$ and nonsignificantly higher than MSI $(p=$ $0.180)$. False-identification rates of the additive coverage lobes were significantly higher than MSI $(p=0.026)$ and EEGfMRI $(p=0.027)$. The intersecting lobes of both methods showed the lowest false identification rate $(13 \% \pm 6 \%, p=$ 0.01).

CONCLUSIONS Both MSI and EEG-fMRI can assist in the presurgical evaluation of patients with refractory epilepsy. The additive use of both tests confers a high identification rate in finding the SL. This combination can help in focusing implantation of icEEG electrodes targeting the SOZ.

https://thejns.org/doi/abs/10.3171/2020.3.JNS192794

KEYWORDS refractory epilepsy; magnetic source imaging; MSI; EEG-fMRI; icEEG; seizure onset zone

$\mathrm{E}$ PILEPSY affects $1 \%$ of the world's population and is mostly well controlled by the use of antiepileptic drugs. ${ }^{1}$ However, $30 \%-40 \%$ of patients fail to respond to trials of two appropriately chosen and well-tolerated medication regimens. ${ }^{2}$ These patients, who suffer from drug-resistant epilepsy (DRE), may benefit from resective epilepsy surgery, which leads to seizure freedom in $40 \%-90 \%$ of cases. ${ }^{3-5}$ Attempts to improve these success rates have focused on appropriate localization of the epileptogenic zone (EZ), which is the main predictive factor for achieving seizure freedom following resective surgery. Intracranial EEG (icEEG) is considered the test of choice for identification of the seizure onset zone (SOZ) before resective surgery, when noninvasive methods have failed. However, the two major pitfalls of icEEG include its invasiveness and the fact that mapping icEEG is limited by electrode position and coverage. ${ }^{6-8}$ Therefore, the development and improvement of noninvasive methods for detecting epileptogenic networks across the brain are the main targets of current research, in order to improve presurgi-

ABBREVIATIONS DRE = drug-resistant epilepsy; $E Z$ = epileptogenic zone; $F C D=$ focal cortical dysplasia; fMRI = functional $M R I ; F P=$ false positive; icEEG = intracranial EEG; IED = interictal epileptiform discharge; MEG = magnetoencephalography; $\mathrm{MSI}$ = magnetic source imaging; $\mathrm{SL}$ = seizure onset lobe; $\mathrm{SOZ}$ = seizure onset zone. SUBMITTED October 13, 2019. ACCEPTED March 6, 2020.

INCLUDE WHEN CITING Published online May 15, 2020; DOI: 10.3171/2020.3.JNS192794.

${ }^{*}$ A.B., N.C., T.G., and I.S. contributed equally to this work. 
cal evaluation of patients with DRE. The two noninvasive methods that are the focus of this article are magnetic source imaging (MSI) and EEG-correlated functional MRI (fMRI).

MSI is a magnetoencephalography (MEG)-derived tool, based on measuring magnetic fields associated with the summated postsynaptic dendritic electric currents. It estimates the position and orientation of the epileptic current dipole by localizing interictal epileptic spikes. ${ }^{9}$ When the current dipole is overlaid onto the patient's coregistered MRI it can assist surgical planning by delineating the EZ. As shown in previous reports, the implementation of MSI in clinical practice resulted in both improved localization of the EZ and seizure outcomes.7,10,11

EEG-fMRI is a more recent method that is based on coupling between interictal epileptiform discharges (IEDs), as measured by scalp EEG, and concurrent brain hemodynamic fluctuations, as recorded by fMRI. ${ }^{12} \mathrm{Al}-$ though clinical evidence supporting the use of this tool continues to be investigated, recent studies have supported its potential role in elucidating epileptic networks and planning resection in DRE cases. ${ }^{13-17}$

In this article we report a retrospective study of patients who underwent both MSI and EEG-fMRI before being operated on for implantation of icEEG monitoring. Our primary aim was to evaluate and compare the capability of each noninvasive method and their combination in correctly identifying the seizure onset lobe (SL).

\section{Methods \\ Patients}

Our study group comprised 14 patients who underwent implantation of icEEG (either subdural grid or depth electrodes) at the Tel-Aviv Medical Center $(\mathrm{n}=12)$ and Hadassah-Jerusalem Medical Center $(n=2)$ during the years 2012-2018 and had a preoperative evaluation that included both MSI and EEG-fMRI.

In each case we collected data regarding preoperative clinical and demographic parameters such as age at epilepsy onset and sex, age at icEEG implantation, hand dominance, type of seizures (focal or generalized), and types of preoperative medications. In addition, we collected data from a battery of preoperative tests, including interictal and video-EEG scalp monitoring, MRI, interictal PET with CT (PET-CT), and neuropsychological evaluation. We then analyzed results of icEEG monitoring regarding the side and lobe of the SOZ, and compared these results with those of MSI and EEG-fMRI. Cases that underwent resection were followed for a period of at least 18 months after surgery, and seizure outcomes by that time were classified according to the Engel classification. Pathology reports of resected surgical specimens were also noted.

\section{Study Groups and Data Analysis}

We compared four types of noninvasive methods for localizing the SL: 1) MSI, 2) EEG-fMRI, 3) MSI and EEGfMRI additive coverage lobes, and 4) MSI and EEG-fMRI intersecting lobes. Results of each noninvasive method were compared to those of icEEG monitoring, which was considered the gold standard for detection of SOZ.

\section{MSI}

MEG recordings were performed in a magnetically shielded room with a whole-head, 248-channel magnetometer array (Magnes $3600 \mathrm{WH}, 4-\mathrm{D}$ Neuroimaging) in Bar-Ilan University, Ramat-Gan, Israel. To suppress distant artifacts, the array of reference coils, located approximately $30 \mathrm{~cm}$ above the head, was employed. The head position was indicated by 5 active coils attached to the scalp. The head position was checked before and after each 10-minute measurement segment. The anatomical landmarks (nasion and preauricular points) and coils were digitized using a Pollhemus FASTTRAK digitizer. The sample rate of measurements was $1017.25 \mathrm{~Hz}$ and the analog online band-pass filter was set on $0.1-400 \mathrm{~Hz}$.

For the MEG data analysis, 4-D Neuroimaging MSI software was used. The magnetic source localization was performed using the spherical head model as a conductor and the single equivalent current dipole model for solving the magnetic field inverse problem. The magnetic field sources were coregistered with anatomical MRI. The magnetic source cluster was defined as 6 or more sources separated by $1 \mathrm{~cm}$ or less. ${ }^{18}$ The source cluster concept in MEG assumes that several different IEDs of the clinically important part of the irritative zone have their sources in the same compact area; this contrasts with the scattered sources, which are much less clinically important. ${ }^{18,19}$ However, using 4-D Neuroimaging MSI software, one IED can be associated with several sources (at different time points), creating an erroneous appearance of the source cluster. Therefore, the data were reviewed by the neurologist (M.M.) to ensure that several IEDs were involved in the source cluster. Only clustered MEG sources were included in the analysis in this paper.

\section{EEG-fMRI}

EEG-fMRI scans were performed using the dual-array EEG method. ${ }^{15}$ MRI scans were performed in two MRI scanners: 6 patients were scanned with a 3.0-T MRI scanner (Signa EXCITE, GE) using a body transmitter coil and an 8-channel head receiver coil, and the other 8 patients with a 3.0-T MRI scanner (Prisma, Siemens) with a 20-channel head receiver coil. A T2*-weighted, gradient echo, echo planar imaging sequence was used for recording the fMRI images. Each scan consisted of 6-8 10-minute recording sessions in which patients were instructed to rest and attempt to sleep. The EEG acquisition protocol and artifact removal were as previously described..$^{15}$ The traces were viewed by a neurologist (M.M.) who manually marked IEDs. These events were used to analyze the fMRI data in an event-related general linear model design using SPM12 (http://www.fil.ion.ucl.ac.uk/spm) and the canonical HRF function with the standard delay. Preprocessing included slice timing correction, 3D motion correction, and coregistration to the anatomical image. The data were smoothed spatially with an 8-mm full-width at half-maximum Gaussian kernel. To account for motion artifacts and outliers, the Artifact Detection Tools (ART) toolbox (https://www.nitrc.org/projects/artifact_detect/) was applied, adding 6 movement regressors as well as an additional regressor for each detected outlier. We chose the 3 clusters with the most significant activation correlating 
with IEDs. These clusters were obtained as follows. First, we started at a stringent false discovery rate corrected T-score threshold that did not include any clusters with a cluster size of 50 voxels or more. Next, we relaxed the threshold until 5 clusters were found (with a T-score above the chosen threshold and a cluster size of at least 50 voxels). These clusters were ordered according to their peak activation in a descending order. In a preliminary analysis we compared the results of the top 1,3 , or 5 clusters and found the best results were obtained for 3 top clusters. Thus, we continued with this result.

MSI and EEG-fMRI additive coverage lobes were defined as the lobes that either MSI or EEG-fMRI identified as SL. MSI and EEG-fMRI intersecting lobes were defined as only those lobes that both MSI and EEG-fMRI identified as SL.

\section{Intracranial EEG Monitoring}

All patients underwent icEEG monitoring after completing their presurgical evaluation, including both MSI and EEG-fMRI examinations. Procedures included implantation of subdural grid or depth electrodes, and patients were then monitored for a few days at the neurological epilepsy unit. Locations of the SL were determined based on identification of the SOZs according to icEEG monitoring. ${ }^{20}$

\section{Statistical Analysis}

To calculate the identification rate of SL by each method, we divided each patient's brain into a total number of 10 potential SLs, 5 on each hemisphere, including frontal, parietal, temporal, occipital, and insular. We then compared the results of each method to those of icEEG monitoring. The identification of a lobe as SL by each method was correct if it was in accordance with icEEG findings.

In each case, the SL identification rate was defined as the number of correctly identified SL by each noninvasive method, divided by the total number of SLs per case according to icEEG. We also measured the false identification rate (false positive [FP]), i.e., the number of healthy lobes according to icEEG that were misclassified by each of the noninvasive methods as SL. The average values of all 14 cases in each test were used to calculate the average identification rates and compare between the tests by applying the Wilcoxon signed-rank nonparametric test. Formal sensitivity and specificity measurements could not be calculated due to the small size of this study and the fact that its reference test (icEEG) does not cover the entire brain, in comparison to both noninvasive methods.

A 2 -sided $p$ value $<0.05$ was considered statistically significant. Statistical analysis was performed using SPSS software (version 21.0, IBM Corp.). This study was approved by the institutional ethics committee of the TelAviv Medical Center.

\section{Results}

During the years 2012-2018, 14 patients with DRE underwent both MSI and EEG-fMRI as part of their preoperative evaluation before implantation of icEEG at the TelAviv Medical Center $(n=12)$ or the Hadassah-Jerusalem
Medical Center $(n=2)$. The mean patient age at icEEG implantation was $23 \pm 9$ years, while the mean age of epilepsy onset was $11 \pm 7$ years. Seven patients $(50 \%)$ were male and 13 (93\%) were right-hand dominant. Eleven patients $(79 \%)$ had no clear localizing findings on preoperative MRI, while only 3 patients had findings suggestive of an epilepsy-related cortical abnormality. Depth electrodes were implanted in 8 patients (57\%), a subdural grid in 5 (36\%), and 1 patient underwent implantation of both depth electrodes and a subdural grid at the same setting. Patients were then followed for an average period of $3.3 \pm 1.9$ years (at least 18 months). Demographic and clinical data in each case are described in Table 1.

\section{MSI, EEG-fMRI, and icEEG}

In reference to icEEG results, MSI had an average correct SL identification rate of $88 \% \pm 31 \%$, similar to that of EEG-fMRI $(73 \% \pm 42 \%)$, showing no statistically significant difference between the two methods $(\mathrm{p}=0.281)$. The FP rate of MSI was $23 \% \pm 7 \%$, comparable to that of EEG-fMRI $(24 \% \pm 6 \%, \mathrm{p}=0.739)$.

\section{MSI and EEG-fMRI Combined Tests and icEEG}

The additive coverage lobes of both MSI and EEGfMRI were able to correctly predict the involved lobe in all cases (i.e., identification rate of $100 \%$ of the SL), significantly higher than EEG-fMRI alone $(p=0.039)$ and nonsignificantly higher than MSI $(p=0.180)$. Their intersecting lobes identified $63 \% \pm 46 \%$ of the SL according to icEEG, which was nonsignificantly lower as compared with MSI and EEG-fMRI ( $\mathrm{p}=0.059$ and $\mathrm{p}=0.180$, respectively). In contrast, FP rates in the additive coverage lobes $(39 \% \pm 8 \%)$ were significantly higher than MSI $(\mathrm{p}=0.026)$ and EEG-fMRI $(\mathrm{p}=0.027)$. As expected, the intersecting lobes showed the lowest FP rate in comparison to the additive method $(13 \% \pm 6 \%, \mathrm{p}=0.01)$.

\section{Postresection Seizure Outcomes}

After completing a battery of preoperative evaluation tests including icEEG monitoring, 10 patients (71\%) underwent craniotomy for resection of the presumed EZ and 1 patient had MRI-guided laser ablation. Eight of the abovementioned procedures (73\%) were extratemporal. Only 3 patients had lesions on MRI. Typical pathological findings of focal cortical dysplasia (FCD) were identified in $9(90 \%)$ of the resected cases, while 1 case showed brain gliosis. After a period of 18 months following surgical intervention, 5 patients $(45 \%)$ were seizure free after surgery (Engel class I), 3 suffered from only rare seizures (Engel II), 1 had a worthwhile improvement (Engel class III), and 2 had no improvement (Engel class IV; Table 1).

\section{Discussion}

In this study, we analyzed the concordance between preoperative mapping of SL by MSI, EEG-fMRI, and their combination in relation to icEEG monitoring in 14 patients. Definitive determination of the SL was based on delineation of the SOZ by icEEG monitoring, which led to resection in 11 patients. ${ }^{20}$ One of the important goals of preoperative investigations is to guide and minimize the 


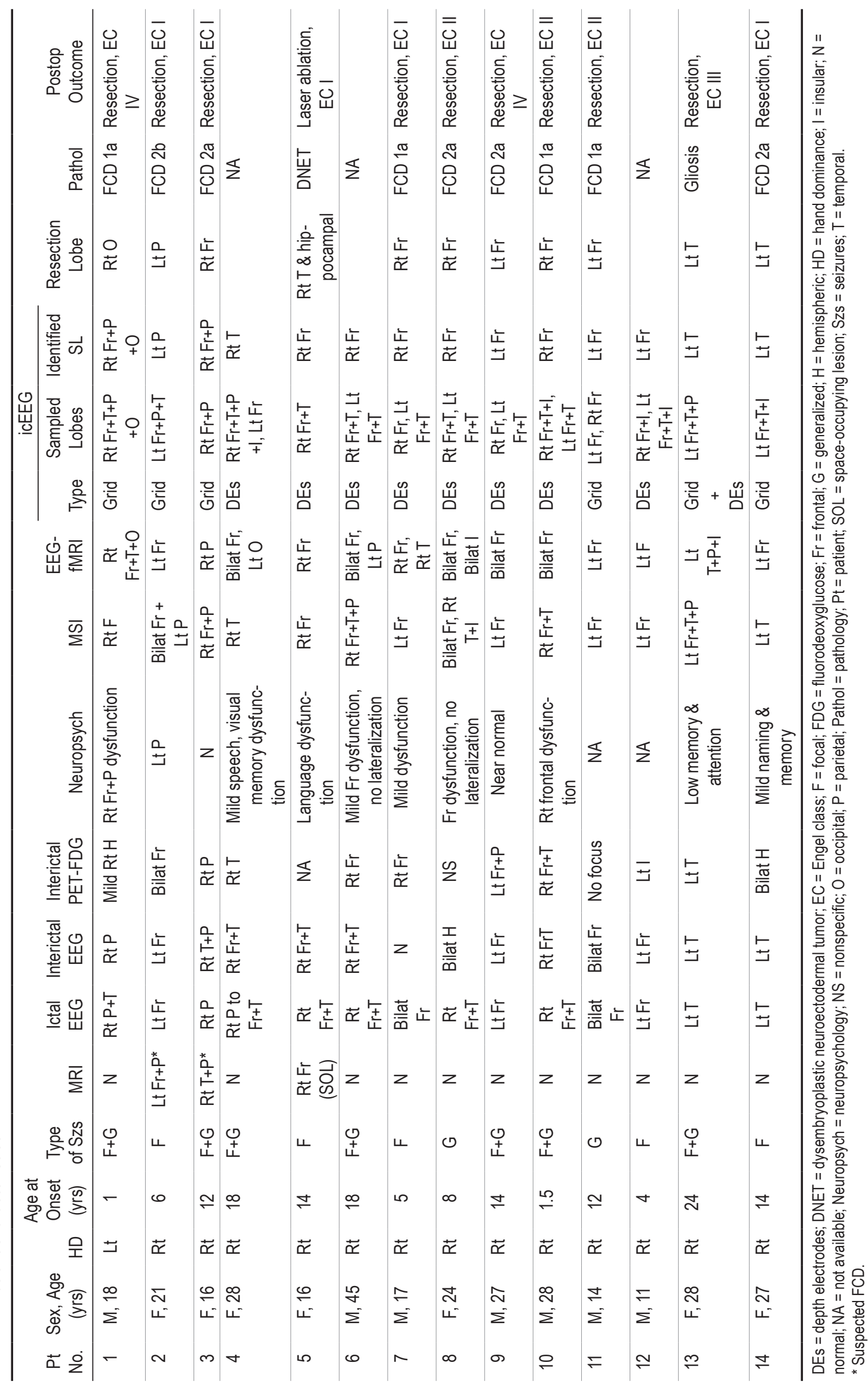



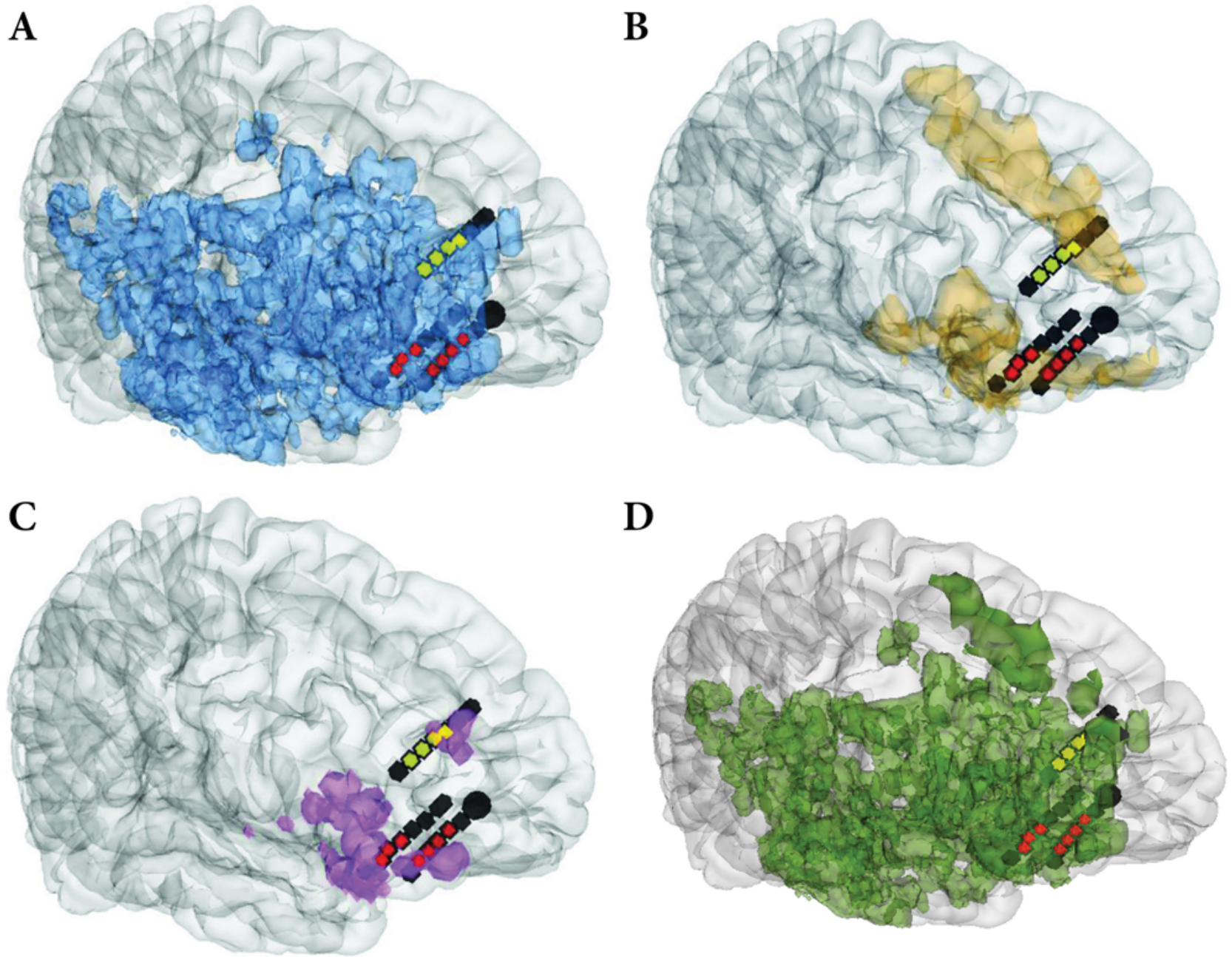

FIG. 1. The MSI dipole map (A, blue), EEG-fMRI activation (B, orange), their intersection (C, magenta), and their additive coverage areas (D, green) of 1 participant are shown on a reconstruction of the right hemisphere (the left hemisphere did not present clustered MEG diploes and only 1 EEG-fMRI cluster in the premotor cortex). Black circles represent 3 relevant depth electrode probes of the 8 inserted. Red and yellow circles mark contacts displaying SOZ activity. The activity in the red contacts was predominant and therefore this area was resected.

number of stereo-EEG electrodes needed to be implanted. According to our results, the combined use of both MSI and EEG-fMRI additive coverage lobes could correctly identify the SL in all cases, and thus can serve as an important tool in guiding stereo-EEG implantation.

MSI alone correctly identified $88 \%$ of the SL. These results are consistent with one of the largest series in the field of MSI and epilepsy by Stefan et al., in which MSI correctly localized the lobe to be treated in $89 \%$ of the 131 surgical cases. ${ }^{21}$ Similarly, our group has previously reported sensitivity and specificity rates of 0.93 and 0.75 , respectively, for source localization by interictal MEG when it was correlated with icEEG in the lobe resolution..$^{10}$ Moreover, several studies have demonstrated the advantages of MSI over ictal and interictal scalp EEG in localizing EZs and supported its role in planning surgical placement of icEEG and resection in DRE., ${ }^{7,22}$ Our results are thus consistent with previous studies regarding the capability of MSI to predict SOZ location.
EEG-fMRI is a more recent method that has been implemented in the presurgical evaluation in a few centers worldwide, mostly under trial conditions. Therefore, sound clinical evidence regarding its role in predicting EZ location is still under investigation. So far, studies that compared interictal activity-related blood oxygen level-dependent (BOLD) signal changes with invasive and noninvasive methods reported a concordance rate of up to $70 \%$ in localizing the EZ at the lobar level. ${ }^{17,23}$ In addition, it was shown that areas of maximal BOLD changes can reach a probability of $>90 \%$ in predicting the location of the EZ..$^{14}$ Furthermore, a higher concordance between preoperative BOLD signal changes and resection area was associated with improved postresection seizure outcomes. ${ }^{17,23}$ Our results, showing SL identification rates of $73 \%$, indicate that EEG-fMRI can have an important role in the preoperative evaluation of patients with DRE.

To our knowledge, currently there has been only one study that directly examined the correlation between MSI 
and EEG-fMRI in localizing interictal epileptic activity. The two methods were found to be concordant, and in certain cases with FCD, this concordance even showed an association with postresection outcomes. ${ }^{24}$ Our study demonstrated relatively similar SL identification rates with either MSI or EEG-fMRI in relation to icEEG findings. These findings support the potential added benefit from simultaneous use of several neuroimaging methods in the presurgical evaluation of patients with DRE. 5,25,26

We studied the combined use of both MSI and EEGfMRI additive coverage lobes in predicting SL locations in relation to icEEG results. We found that the combined additive method could correctly identify as much as $100 \%$ of the icEEG-defined SL, significantly higher than EEGfMRI alone and nonsignificantly higher than MSI. However, this method was also characterized by a higher FP rate than either MSI or EEG-fMRI alone. Conversely, the intersecting lobes showed the lowest FP rate of $13 \% \pm 6 \%$.

We suggest that the high identification rate of the additive coverage lobes may assist surgical planning by sparing the implantation of icEEG in lobes outside this area, which had a low probability for epileptic activity. In contrast, the intersecting lobes method could focus the surgeon on lobes that are more likely to contain the SOZ. Figure 1 explicitly describes the abovementioned findings in one of our study cases.

Furthermore, we looked at the 1.5-year clinical followup of 11 patients who underwent resection or ablation for epilepsy. Seizure freedom (Engel class I) was achieved in $5(45 \%)$ of 11 patients during the abovementioned period. These results are comparable to other series with a high rate of extratemporal epilepsies, especially in MRI-negative cases as in our cohort. ${ }^{5,27-29}$ Epilepsy-related pathological findings were found in all of the resected cases, supporting the correct identification of the SOZ by icEEG and its use as a reference. ${ }^{30-34}$ Extratemporal surgeries are known to be limited by eloquent boundaries and are linked to lower rates of seizure freedom, ranging between $21 \%$ and $61 \%$ in frontal lobe resections, for example..$^{5,27-29}$ Therefore, noninvasive methods such as MSI and EEGfMRI are not accurate enough on their own to guide resection, rather they can assist in predicting the involved lobe and guide the implantation of intracranial electrodes for definitive identification of the SOZ.

\section{Limitations}

The main limitation of this study is its small size and low power, which affected the chances of drawing sound and significant conclusions. We thus could not perform a subgroup analysis in terms of postresection seizure outcomes and we are currently working on larger series that will be able to address such issues. Furthermore, this work focused only on analysis at the lobar level. Due to the small sample size we could not reach extensive electrode sampling to evaluate the results in higher resolutions that might delineate the SOZ in the sublobar level. It is possible that at a higher resolution, EEG-fMRI would show more favorable outcomes than MSI in predicting the SOZ location, considering its higher spatial resolution. ${ }^{10,12,17} \mathrm{We}$ therefore believe that larger studies are imperative in order to shed more light on these concepts. In addition, one may argue that the correlation between both noninvasive tests and icEEG derived from their influence on icEEG implantation.

MSI and EEG-fMRI guided the targeting process of icEEG in this study, yet they were used in combination with other parameters, including the clinical semiology, neuropsychological analysis, scalp EEG, video-EEG, and PET-CT. Together with the fact that all pathological specimens showed epilepsy-related findings, we believe icEEG results can be used as a reference to MSI and EEG-fMRI results.

\section{Conclusions}

Both MSI and EEG-fMRI may improve preoperative planning of resection in patients with DRE. As both tests are interictal, they can be easily incorporated into the presurgical workup, as opposed to ictal studies. The combined use of both tests' entire coverage lobes may confer high identification rates of the presumed SOZ locations and can therefore assist preoperative planning by directing the implantation of icEEG monitoring.

\section{References}

1. World Health Organization. Newsroom Fact Sheets. Epilepsy. June 20, 2019. Accessed March 18, 2020. https://www.who. int/news-room/fact-sheets/detail/epilepsy

2. Kwan P, Sperling MR. Refractory seizures: try additional antiepileptic drugs (after two have failed) or go directly to early surgery evaluation? Epilepsia. 2009;50(suppl 8):57-62.

3. Engel J Jr. The current place of epilepsy surgery. Curr Opin Neurol. 2018;31(2):192-197.

4. Engel J Jr. What can we do for people with drug-resistant epilepsy? The 2016 Wartenberg Lecture. Neurology. 2016;87(23):2483-2489.

5. Vakharia VN, Duncan JS, Witt JA, et al. Getting the best outcomes from epilepsy surgery. Ann Neurol. 2018;83(4):676690.

6. Englot DJ, Han SJ, Rolston JD, et al. Epilepsy surgery failure in children: a quantitative and qualitative analysis. $J$ Neurosurg Pediatr. 2014;14(4):386-395.

7. Englot DJ, Nagarajan SS, Imber BS, et al. Epileptogenic zone localization using magnetoencephalography predicts seizure freedom in epilepsy surgery. Epilepsia. 2015;56(6):949-958.

8. Englot DJ, Raygor KP, Molinaro AM, et al. Factors associated with failed focal neocortical epilepsy surgery. Neurosurgery. 2014;75(6):648-656.

9. Hari R, Salmelin R. Magnetoencephalography: from SQUIDs to neuroscience. Neuroimage 20th anniversary special edition. Neuroimage. 2012;61(2):386-396.

10. Medvedovsky M, Taulu S, Gaily E, et al. Sensitivity and specificity of seizure-onset zone estimation by ictal magnetoencephalography. Epilepsia. 2012;53(9):1649-1657.

11. Wilenius J, Medvedovsky M, Gaily E, et al. Interictal MEG reveals focal cortical dysplasias: special focus on patients with no visible MRI lesions. Epilepsy Res. 2013;105(3):337348.

12. Vulliemoz S, Lemieux L, Daunizeau J, et al. The combination of EEG source imaging and EEG-correlated functional MRI to map epileptic networks. Epilepsia. 2010;51(4):491505.

13. Bénar CG, Grova C, Kobayashi E, et al. EEG-fMRI of epileptic spikes: concordance with EEG source localization and intracranial EEG. Neuroimage. 2006;30(4):1161-1170.

14. Khoo HM, Hao Y, von Ellenrieder N, et al. The hemodynamic response to interictal epileptic discharges localizes the seizure-onset zone. Epilepsia. 2017;58(5):811-823. 
15. Klovatch-Podlipsky I, Gazit T, Fahoum F, et al. Dual array EEG-fMRI: an approach for motion artifact suppression in EEG recorded simultaneously with fMRI. Neuroimage. 2016;142:674-686.

16. Liu JV, Kobylarz EJ, Darcey TM, et al. Improved mapping of interictal epileptiform discharges with EEG-fMRI and voxel-wise functional connectivity analysis. Epilepsia. 2014;55(9):1380-1388.

17. van Graan LA, Lemieux L, Chaudhary UJ. Methods and utility of EEG-fMRI in epilepsy. Quant Imaging Med Surg. 2015;5(2):300-312.

18. Iida K, Otsubo H, Matsumoto Y, et al. Characterizing magnetic spike sources by using magnetoencephalography-guided neuronavigation in epilepsy surgery in pediatric patients. $J$ Neurosurg. 2005;102(2)(suppl):187-196.

19. Ramachandran VS, Altschuler EL. The use of visual feedback, in particular mirror visual feedback, in restoring brain function. Brain. 2009;132(Pt 7):1693-1710.

20. Rosenow F, Lüders H. Presurgical evaluation of epilepsy. Brain. 2001;124(Pt 9):1683-1700.

21. Stefan H, Hummel C, Scheler G, et al. Magnetic brain source imaging of focal epileptic activity: a synopsis of 455 cases. Brain. 2003;126(Pt 11):2396-2405.

22. Agarwal N, Krishnan B, Burgess RC, et al. Magnetoencephalographic characteristics of cortical dysplasia in children. Pediatr Neurol. 2018;78:13-19.

23. Thornton R, Laufs H, Rodionov R, et al. EEG correlated functional MRI and postoperative outcome in focal epilepsy. J Neurol Neurosurg Psychiatry. 2010;81(8):922-927.

24. Heers M, Hedrich T, An D, et al. Spatial correlation of hemodynamic changes related to interictal epileptic discharges with electric and magnetic source imaging. Hum Brain Mapp. 2014;35(9):4396-4414.

25. Pittau F, Grouiller F, Spinelli L, et al. The role of functional neuroimaging in pre-surgical epilepsy evaluation. Front Neurol. 2014;5:31.

26. Vaudano AE, Carmichael DW, Salek-Haddadi A, et al. Networks involved in seizure initiation. A reading epilepsy case studied with EEG-fMRI and MEG. Neurology. 2012;79(3):249-253.

27. Bulacio JC, Jehi L, Wong C, et al. Long-term seizure outcome after resective surgery in patients evaluated with intracranial electrodes. Epilepsia. 2012;53(10):1722-1730.
28. Lazow SP, Thadani VM, Gilbert KL, et al. Outcome of frontal lobe epilepsy surgery. Epilepsia. 2012;53(10):1746-1755.

29. Malmgren K, Edelvik A. Long-term outcomes of surgical treatment for epilepsy in adults with regard to seizures, antiepileptic drug treatment and employment. Seizure. 2017:44:217-224.

30. Blumcke I, Spreafico R, Haaker G, et al. Histopathological findings in brain tissue obtained during epilepsy surgery. $N$ Engl J Med. 2017;377(17):1648-1656.

31. Cohen-Gadol AA, Ozduman K, Bronen RA, et al. Long-term outcome after epilepsy surgery for focal cortical dysplasia. $J$ Neurosurg. 2004;101(1):55-65.

32. Fauser S, Essang C, Altenmüller DM, et al. Long-term seizure outcome in 211 patients with focal cortical dysplasia. Epilepsia. 2015;56(1):66-76.

33. Lee SK, Kim DW. Focal cortical dysplasia and epilepsy surgery. J Epilepsy Res. 2013;3(2):43-47.

34. Tassi L, Colombo N, Garbelli R, et al. Focal cortical dysplasia: neuropathological subtypes, EEG, neuroimaging and surgical outcome. Brain. 2002;125(Pt 8):1719-1732.

\section{Disclosures}

Dr. Medvedovsky reports being one of the authors of the patent related to the dual-array EEG-fMRI approach.

\section{Author Contributions}

Conception and design: Berger, Cohen, Fahoum, Medvedovsky, Fried, Gazit, Strauss. Acquisition of data: Berger, Cohen, Medvedovsky, Meller, Ekstein, Benifla, Fried, Gazit, Strauss. Analysis and interpretation of data: Berger, Cohen, Fahoum, Medvedovsky, Aizenstein, Fried, Gazit, Strauss. Drafting the article: Berger, Cohen, Fahoum, Medvedovsky, Ekstein, Benifla, Aizenstein, Fried, Gazit, Strauss. Statistical analysis: Berger. Study supervision: Fried, Gazit, Strauss.

\section{Correspondence}

Assaf Berger: Tel Aviv Medical Center, Tel Aviv, Israel. assaf. berger@gmail.com. 